\title{
On the Performance of the Zoned Fishnet Metamaterial Lens with Positive and Negative Reference Phase
}

\author{
V. Pacheco-Peña, Student Member, IEEE, I. V. Minin, O. V. Minin and M. Beruete
}

\begin{abstract}
In this paper, several converging fishnet metalenses are designed using both the zoning and reference phase techniques. They are engineered with a focal length of $1.5 \lambda_{0}$ at the design frequency of $55 \mathrm{GHz}\left(\lambda_{0}=5.45 \mathrm{~mm}\right)$ at which the fishnet metamaterial has an effective refractive index of $n_{\text {lens }}=$ 0.68. Three metalenses are analyzed: with positive, negative and without reference phase in order to compare their performance. The focal properties are evaluated both numerically and experimentally, demonstrating a good agreement between them. The best performance is achieved for the zoned metalens with positive reference phase, with an experimental power enhancement at the focal length of $6.2 \mathrm{~dB}$, better axial resolution $\left(0.65 \lambda_{0}\right)$ and reduced lateral lobes compared to the other designs.
\end{abstract}

Index Terms-Fresnel zoning, zoned lenses, fishnet metamaterial, millimeter-waves.

\section{INTRODUCTION}

$\mathrm{M}$ ETAMATERIALS have become an important field of research because they enable a full control of the electromagnetic response[1]. They have given rise to metadevices (metamaterial-based devices) that exhibit an improved performance compared to those made with natural materials[2]. For instance, improved directional antennas[3], [4], sensors[5], nanocircuits[6] and cloaking[7], [8] are just a few fields where metamaterials-based devices have been demonstrated to surpass the performance of devices made with natural materials, in a wide spectral range ranging from microwave to optical frequencies.

Lenses have also greatly benefited from metamaterials research. Different solutions have been reported depending on

Manuscript received XX, 2016. We would like to thank Dr. Victor Torres for the fabrication of the prototypes. This work was supported by the Spanish Ministerio de Economía y Competitividad under contract TEC2014-51902C2-2-R and partially by the Tomsk State University Competitiveness Improvement Program $\mathrm{N}^{\mathrm{o}}$ 8.1.23.2015. V.P.-P.is sponsored by Spanish Ministerio de Educación, Cultura y Deporte under grant FPU AP-2012-3796. M.B. is sponsored by the Spanish Ministerio de Economía y Competitividad via RYC-2011-08221.

V. Pacheco-Peña and M. Beruete are with Antennas Group-TERALAB, Universidad Pública de Navarra, Pamplona 31006, Spain (e-mail: victor.pacheco@unavarra.es, miguel.beruete@unavarra.es). M. Beruete is also with the Institute of Smart Cities, Public University of Navarra.

O.V. Minin is with the National Research Tomsk State University, Lenina Ave. 36, Tomsk 634050, Russia (e-mail: prof.minin@gmail.com). I.V.Minin is with the Tomsk Politechnical University. the application and spectral window to be used such as lenses based on transformation optics[9], graded-index technique[10] and negative refractive index (NRI) media[11]. Within this realm, the fishnet metamaterial with double in-plane periodicity has been applied in the design of all metallic metalenses within the millimeter-wave spectrum[12] due to its low loss at high frequencies. This type of artificial material is also known as extraordinary transmission metamaterial[13] since it consists of stacked subwavelength hole arrays working below cut-off of the holes, where high transmission peaks and NRI are obtained[14].

Recently, the zoning technique has been applied in the design of fishnet metalenses to reduce their volume[12]. This technique consists of sequentially removing parts of the metalens when the phase variation (with respect to free-space) of the waves traveling through them is an integer multiple of $2 \pi[15]$. It has been shown that the overall performance of the zoned fishnet metalens is not deteriorated when using this technique compared with the full-profiled designs[16], [17]. To improve their performance in terms of side lobe reduction (an additional parameter to the phase quantization levels) the reference phase technique [18] (borrowed from Fresnel Zone Plate (FZP) lenses) has been recently applied along with the zoning procedure to reduce the side lobes and increase the power at the focal length $(F L)[19]$, in good agreement with FZP lenses[18].

Inspired by the success of translating the zoning and reference phase techniques to the design of fishnet metalenses, in this work we show the design, numerical and experimental results of several zoned fishnet metalenses with positive and negative values of reference phase. Unlike our previous work where positive values of reference phase were used[19], here we further extend the study of this technique and three metalenses are designed: with positive, negative and without reference phase to compare their focal properties. A small $F L$ is chosen $\left(1.5 \lambda_{0}\right)$ at the design frequency of $55 \mathrm{GHz}\left(\lambda_{0}=\right.$ $5.45 \mathrm{~mm}$ ), where the fishnet metamaterial has a refractive index of $n_{\text {lens }} \sim 0.68$, to get a compact antenna lens system.

\section{DESIGN}

As described in our previous works[16], [17], the zoning technique is based on the reduction of the profile of the lens each time a phase advance of $2 \pi$ is reached inside the material (with respect to free-space propagation).This phase advance is 
directly related to the thickness of the lens. Hence, its profile is reduced each time a thickness limit $\left[t=\lambda_{0} /\left(1-n_{\text {lens }}\right)\right.$, where $n_{\text {lens }}$ is the refractive index of the material of the lens] is reached. Therefore, unlike planar FZP lenses (where a reference phase from 0 to $\pi$ is applied), the extra phase advance introduced with the reference phase for zoned lenses may be modulated from 0 to $2 \pi$, so that the total phase advance is between 0 and $4 \pi$. This implies an increase of the thickness of each zone that can be calculated mathematically as follows[19]:

$$
\Delta t=q t=q \frac{\lambda_{0}}{1-n_{\text {lens }}}
$$

where $\Delta t$ is the extra thickness introduced by the reference phase and $q$ is a factor between 0 and 1 corresponding to an extra phase advance (from 0 to $2 \pi$ ). Therefore, when the reference phase is combined with the zoning technique, the lens profile is stepped when a thickness $t_{\text {total }}=t \pm \Delta t$ is reached. Finally, the zoned profile can be designed by using Eq. (1) and $t_{\text {total }}$ along with the well-known expression of a conical section[12], as follows:

$$
z=\bmod \left[\frac{F L\left(1-n_{\text {lens }}\right)-\sqrt{F L^{2}\left(1-n_{\text {lens }}\right)^{2}-x^{2}\left(1-n_{\text {lens }}^{2}\right)}}{\left(1-n_{\text {lens }}^{2}\right)}, t_{\text {total }}=t(1 \pm q)\right]
$$

where mod is the modulus operation. Note that in the above equation, the factor $q$ can be positive or negative. In the following, we will describe the design process of several zoned fishnet metalenses using Eqs. (1-2) and considering positive and negative values of $q$ with the aim to evaluate their focusing properties.

The unit cell used for the fishnet metamaterial is shown in the inset of Fig. 1(a). It has the next dimensions: $w=0.35 \mathrm{~mm}$, $d_{x}=3 \mathrm{~mm}, d_{y}=5 \mathrm{~mm}, d_{z}=1.35 \mathrm{~mm}$ and $a=2.4 \mathrm{~mm}$. Copper $\left(\sigma_{\mathrm{Cu}}=5.8 \times 10^{7} \mathrm{~S} / \mathrm{m}\right)$ is used as metal. With these parameters, a double in-plane unit cell is used which has been demonstrated to filter the cross-polarization[20]. Notice that the unit cell has been changed from those used in previous works[12], [16], [17], [19]for fabrication purposes. With these parameters, the eigenmode solver of the commercial software CST Microwave Studio ${ }^{\circledR}$ is used to retrieve the refractive index of the infinitely replicated unit cell. The results are shown in Fig. 1(a). From this figure the dispersive performance of the fishnet metamaterial is evident, showing a NRI band, as expected.

As in the previous studies[16], [19], the near-zero refractive index condition is selected in the design of the metalenses to work within the region where the refractive index of the fishnet metamaterial has a smooth variation. However, by inspecting Eq. (2) one can find that by reducing the value of $\left|n_{\text {lens }}\right|$, the first zone will be thicker due to the thickness limit $\left(t_{\text {total }}\right)$ condition. Since a small $F L$ is used in the design, the first zone would be closer (just a few millimeters) to the center of the metalens $(x=y=0)$ which may difficult the experimental characterization of the focal properties. Additionally, we also want to avoid the frequencies where the values of $n_{\text {lens }}$ are close to -1 since the value of $n_{\text {lens }}$ changes

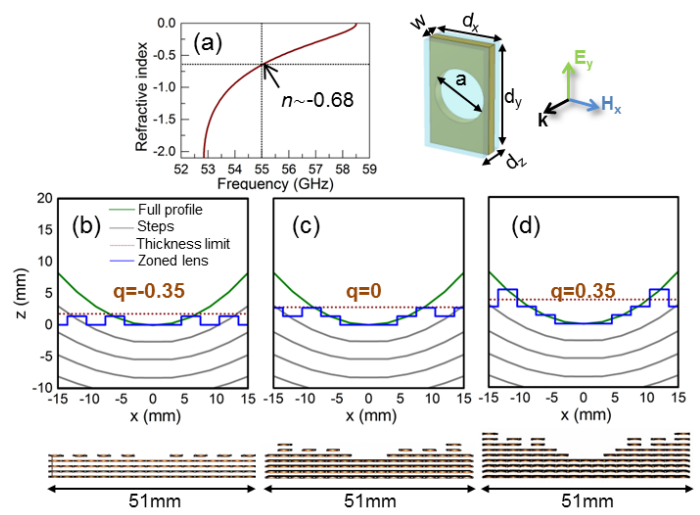

Fig. 1. (a) Effective refractive index for a metamaterial made of an infinite number of stacked subwavelength holes (left). (Right) Schematic representation of the unit cell with parameters: $d_{x}=3 \mathrm{~mm}, d_{y}=5 \mathrm{~mm}, d_{z}=$ $1.35 \mathrm{~mm}, a=2.4 \mathrm{~mm}$ and metal thickness $w=0.35 \mathrm{~mm}$. Calculated profile of the metalenses with: (b) $q=-0.35$, (c) $q=0$ and (d) $q=0.35$. Each curve represents: the full concave profile (green curve), successive steps (grey lines), thickness limit (red dotted line) and the zoned profile (blue curve). A top view of each profile using the fishnet metamaterial is shown at the bottom of panels (b-c). The center (origin) of the metalenses is defined at $x$ $=y=z=0 \mathrm{~mm}$. The focal length is the distance from the origin of the metalenses where the maximum power is achieved.

abruptly within this region [as observed in Fig. 1(a)] and could result in a strong spectral deviation of the focus from the designed value. Therefore, as a trade-off between these two conditions, the design frequency is chosen at $55 \mathrm{GHz}\left(\lambda_{0}=\right.$ $5.45 \mathrm{~mm}$ ) at which the effective refractive index of the fishnet metamaterial is $n_{\text {lens }} \sim-0.68$. Taking into account these factors, three zoned fishnet metalenses are designed: i) with negative $(q=-0.35)$, ii) positive $(q=0.35)$ and iii) without reference phase $(q=0)$ in order to compare their performance. The resulting final profiles are shown in Fig. 1(b-d). Here, cylindrical metalenses are designed to ease their fabrication. It is important to highlight that here only three discrete cases of $q$ are used. This is because of the fishnet metamaterial has fixed dimensions along the propagation $z$ axis [see Fig. 1(a)]. Hence, only discrete values of $q$ are possible for this unit cell[19].

\section{NUMERICAL AND EXPERIMENTAL RESULTS}

The focusing performance of the metalenses is evaluated both numerically and experimentally. The numerical simulations are carried out using the transient solver of the commercial software CST Microwave Studio ${ }^{\circledR}$ following the same procedure as in our previous works[16], [19]. First, an evaluation of the structures is performed in terms of frequency and $F L$. For this purpose, the complete cylindrical metalenses are simulated with a total number of $17 \times 13$ holes along the $x$ and $y$ axis, respectively, with total dimensions of $9.35 \lambda_{0} \times 11.91 \lambda_{0}$. The central part of the metalenses has four perforated plates, two more than in our previous designs[16], [19]. This is because we are working within the limit where $n_{\text {lens }}$ has abrupt or smooth variations (as discussed before). Therefore, more metal plates are required to obtain a similar performance to the ideal infinite fishnet metamaterial. Finally, electric and magnetic probes are also defined along the 


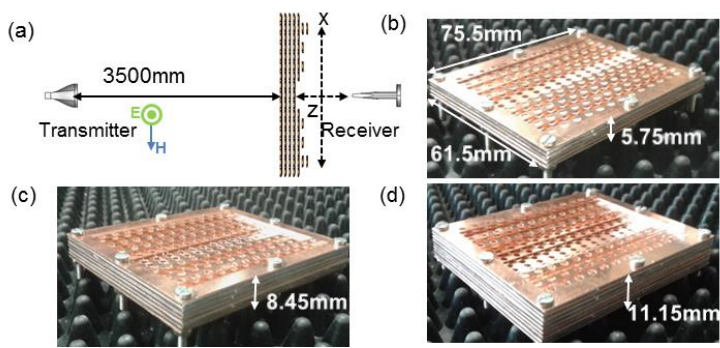

Fig. 2. Schematic representation of the experimental setup used to characterize the focusing properties of the zoned fishnet metalenses. Photographs of the fabricated prototypes with: (b) $q=-0.35$, (c) $q=0$ and (d) $q=0.35$. The metalenses are fabricated with stacked perforated plates attached with screws using an assembly frame. The air gap between plates consists of a frame with a thickness of $1 \mathrm{~mm}$.

propagation $z$ axis at $x=y=0$ (i.e., the center of the metalenses) from $z=3 \mathrm{~mm}$ to $z=20 \mathrm{~mm}$ with a step of 0.2 $\mathrm{mm}$ to record the field distribution as a function of frequency.

For the experiment, an $\mathrm{ABmm}^{\mathrm{TM}}$ Quasi-Optical Vector Network analyzer is used working in the V-band of millimeter waves. A schematic representation of the experimental setup is shown in Fig. 2(a). The metalenses are placed in between a high gain horn antenna located far $(3500 \mathrm{~mm})$ from their flat face (to illuminate them with a planewave) and an open ended probe which is used as a receiver. The probe is placed on a translation stage to record the electric field distribution on the focal plane. Similarly to the numerical study, we first evaluate the spectral response of the metalenses to find the position of the focus. The pictures of the fabricated prototypes and their final dimensions are shown in Fig. 2(b-d) for the designs with $q=-0.35, q=0$ and $q=0.35$, respectively.

The numerical and experimental results of the normalized power distribution spectra along the propagation $z$ axis are shown in Fig. 3(a,c,e) and Fig. 3(b,d,f), respectively, for each metalens. In general terms, the experimental results are in a relative good agreement with the numerical simulations in terms of the frequency where the maximum power is obtained. In the latter case, the peak of power occurs at $55 \mathrm{GHz}$ for all the designs, which corresponds to the design frequency. From the experimental values, the maximum happens at the frequency of $55.5 \mathrm{GHz}\left(\lambda_{0}=5.4 \mathrm{~mm}\right)$, i.e. a very small error of $0.9 \%$ from the designed value, which may be due to experimental misalignments and fabrication tolerances. From here on, these frequencies are used for the numerical and experimental study, respectively.

From the simulations, the $F L$ of each metalens is $F L_{\text {sim }} q=-$ $0.35=8.2 \mathrm{~mm}\left(1.5 \lambda_{0}\right), F L_{\text {sim } \_q=0}=8.71 \mathrm{~mm}\left(1.59 \lambda_{0}\right), F L_{s i m_{-} q=0.35}$ $=8.6 \mathrm{~mm}\left(1.57 \lambda_{0}\right)$ and from the experiment, they are $F L_{\text {exp }} q=-$ $0.35=8.4 \mathrm{~mm}\left(1.55 \lambda_{0}\right), F L_{\text {exp_q } q 0}=8.5 \mathrm{~mm}\left(1.57 \lambda_{0}\right), F L_{\text {exp } \_q=0.35}$ $=8.5 \mathrm{~mm}\left(1.57 \lambda_{0}\right)$. As observed, the $F L$ is close to the designed value of $8.18 \mathrm{~mm}\left(1.5 \lambda_{0}\right)$ for all the designs, demonstrating the good agreement between the numerical, experimental and designed values. For the sake of completeness, the numerical and experimental results of the power distribution at each $F L$ as a function of frequency are shown in Fig. 4(a,b), respectively, where again it is clear the agreement between both results.

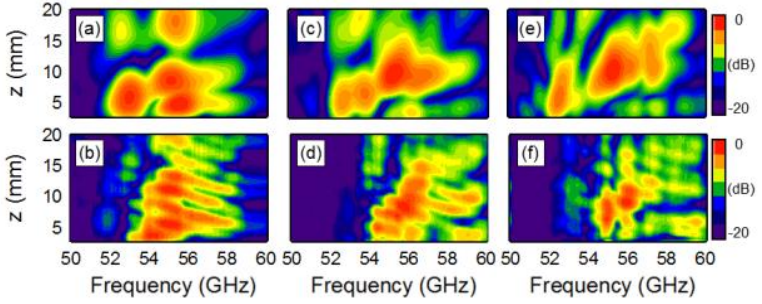

Fig. 3. Simulation (first row) and experimental (second row) results of the normalized power distribution spectra along the propagation $z$-axis at $x=0$ $\mathrm{mm}$ from the center of the metalenses for the designs with a factor of reference phase: $q=-0.35(\mathrm{a}, \mathrm{b}), q=0(\mathrm{c}, \mathrm{d})$ and $q=0.35(\mathrm{e}, \mathrm{f})$.

After the spectral response has been studied, the focusing performance of the fishnet metalenses is evaluated at the operation frequency. The results of the power distribution on the focal plane $(x z)$ are shown in the first and second row of Fig. 5, respectively, for the cases of the designs with $q=0$ (first column), $q=0.35$ (second column) and $q=-0.35$ (third column). Again, there is a good agreement between the numerical and the experimental results. Moreover, a clear focus is obtained for the designs with $q=0$ and $q=0.35$ whereas the focus is strongly deteriorated when a negative reference phase is used $(q=-0.35)$. This is due to the fact that, with the latter design, the metalens only has one zoned plate [see Fig. 1(b) and Fig. 2(b)]. Hence, the profile of the lens is almost a single grating which produces a focus with strong lateral lobes.

To better compare these results, the numerical and experimental results of the power distribution along the $x$ axis at each $F L$ for the three designs are plotted in Fig. 5(g,h), respectively. A good agreement is observed between these results. Note that the lateral lobes are reduced when using a positive reference phase in both numerical and experimental results, in a good agreement with the designs discussed in a previous work[19]. When a negative value is introduced, the lateral lobes are increased due to the influence of only one zone, as explained before. A summary of the focusing properties are shown in Table I in terms of the $F L$, full-width at half-maximum along the transversal $x$ axis $\left(F W H M_{x}\right)$, depth of focus $D F$ (defined as the distance at which the power distribution at the $F L$ has decayed half of its maximum along the axial $z$ axis) and power enhancement. As shown there, the best performance is achieved when $q=0.35$ in terms of the $F L, D F$ and power enhancement, as happened in the metalenses we studied before with a $F L$ of $4.5 \lambda_{0}[19]$. Also, note that the power enhancement for these metalenses is below
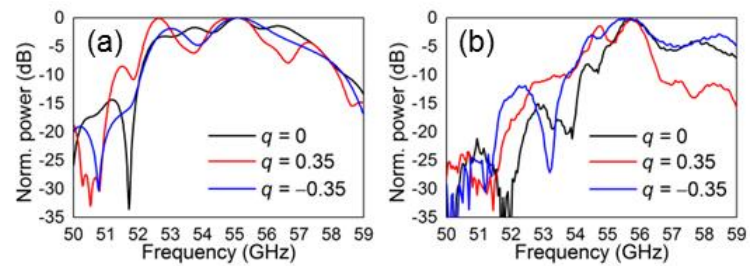

Fig. 4. Simulation (a) and experimental (b) results of the normalized power distribution spectra at each $F L$ for the designs with $q=-0.35$ (blue), $q$ $=0$ (black) and $q=0.35$ (red). 

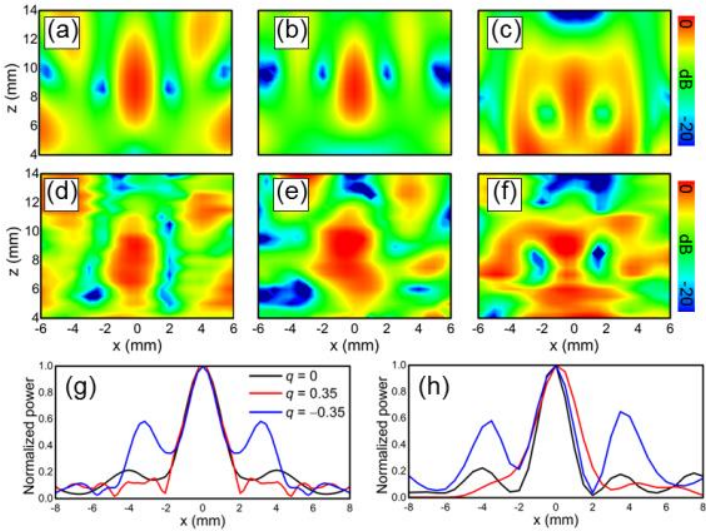

Fig. 5. Simulation (first row) and experimental (second row) results of the normalized power distribution in the $x z$-plane for the metalenses with $q=0$ (first column), $q=0.35$ (second column) and $q=-0.35$ (third column) at the numerical and experimental frequency of $55 \mathrm{GHz}$ and $55.5 \mathrm{GHz}$, respectively. Numerical $(\mathrm{g})$ and experimental (h) results of the power distribution along the transversal $x$ axis at each FLfor the designs with $q=0$ (black), $q=0.35$ (red) and $q=-0.35$ (blue).

the values obtained with our previous reported fishnet metalenses[16], [17] and other advanced designs[21], due to the fact that more plates are used at their center, increasing the loss in the structures. However, these results demonstrate that the reference phase can be also applied in the design of metalenses with small $F L$ obtaining the best performance for positive values of $q$, reducing the lateral lobes at the focal position. Note that this performance is valid for the metalenses here studied. As described before, because of the discretization of the fishnet metamaterial along the $z$ axis, we can only evaluate one case of negative reference phase. If a metamaterial with smaller thickness is used, different negative values of $q$ may be applied which could lead to an improved performance of a metalens even using values of $q<0$.

TABLE I

FOCUSING PERFORMANCE OF THE METALENSES

\begin{tabular}{ccccccc}
\hline \hline & \multicolumn{2}{c}{$q=0$} & \multicolumn{2}{c}{$q=0.35$} & \multicolumn{2}{c}{$q=-0.35$} \\
\hline & Sim & Exp & Sim & Exp & Sim & Exp \\
\hline$F L^{\mathrm{a}}\left(\lambda_{0}\right)$ & 1.59 & 1.57 & 1.57 & 1.57 & 1.5 & 1.55 \\
$F W H M_{x}{ }^{\mathrm{b}}\left(\lambda_{0}\right)$ & 0.38 & 0.33 & 0.37 & 0.39 & 0.41 & 0.35 \\
$D F^{\mathrm{c}}\left(\lambda_{0}\right)$ & 1.01 & 0.83 & 0.96 & 0.65 & 1.19 & 0.92 \\
Enh. $(\mathrm{dB})$ & 6.3 & 6 & 6.4 & 6.2 & 2.6 & 2.5 \\
\hline \hline
\end{tabular}

The numerical and experimental values are given at the frequency of 55 $\mathrm{GHz}$ and $55.5 \mathrm{GHz}$, respectively.

${ }^{\mathrm{a}} \mathrm{FL}$ is the Focal Length

${ }^{b} \mathrm{FWHM}_{\mathrm{x}}$ is the Full-Width at Half-Maximum

${ }^{c} \mathrm{DF}$ is the Depth of Focus

\section{CONCLUSION}

Zoned fishnet metalenses with positive and negative values of reference phase has been presented. The focusing properties of three metalenses (with positive, negative and without reference phase) have been evaluated both numerically and experimentally with a good agreement between them. The best performance in terms of the $F L, F W H M_{x}, D F$ and power enhancement has been achieved with a positive reference phase. When a negative reference phase is used, the lateral lobes at the $F L$ are strongly increased due to the fact that only one zoned plate is used in the prototype. Therefore, the focal plane of this metalens is strongly deteriorated compared with the case without and with positive reference phase. The prototypes here proposed may be applied to integrated and compact solutions where reduced lateral lobes and small distances of the focus are needed.

\section{REFERENCES}

[1] N. Engheta and R. Ziolkowski, Metamaterials:Physics and Engineering Explorations, 1st ed. USA: John Wiley \& Sons \& IEEE Press, 2006.

[2] N. I. Zheludev and Y. S. Kivshar, "From metamaterials to metadevices," Nat. Mater., vol. 11, no. 11, pp. 917-924, 2012.

[3] S. Enoch, G. Tayeb, P. Sabouroux, N. Guerin, and P. Vincent, "A metamaterial for directive emission," Phys. Rev. Lett., vol. 89, no. 21, p. 213902, Nov. 2002.

[4] A. Ourir and a De Lustrac, "Metamaterial-based phased array for directional beam steering," Microw. Opt. Technol. Lett., vol. 51, no. 11, pp. 2653-2656, 2009.

[5] A. Alù and N. Engheta, "Dielectric Sensing in $\epsilon$-Near-Zero Narrow Waveguide Channels," Phys. Rev. B, vol. 78, no. 4, p. 15, 2008.

[6] N. Engheta, "Circuits with light at nanoscales: optical nanocircuits inspired by metamaterials.," Science, vol. 317, no. 5845, pp. 1698-702, Sep. 2007.

[7] N. Landy and D. R. Smith, "A full-parameter unidirectional metamaterial cloak for microwaves," Nat. Mater., vol. 12, pp. 25-28, 2013.

[8] B. Orazbayev, N. Mohammadi Estakhri, M. Beruete, and A. Alù, "Terahertz carpet cloak based on a ring resonator metasurface," Phys. Rev. B, vol. 91, no. 19, p. 195444, 2015.

[9] A. Demetriadou and Y. Hao, "A Grounded Slim Luneburg Lens Antenna Based on Transformation Electromagnetics," IEEE Antennas Wirel. Propag. Lett., vol. 10, pp. 1590-1593, 2011.

[10] R. B. Greegor, C. G. Parazzoli, J. A. Nielsen, M. A. Thompson, M. H. Tanielian, and D. R. Smith, "Simulation and testing of a graded negative index of refraction lens," Appl. Phys. Lett., vol. 87, no. 9, pp. 91114-1-3, 2005.

[11] J. B. Pendry, "Negative refraction makes a perfect lens," Phys. Rev. Lett., vol. 85, no. 18, pp. 3966-3969, 2000.

[12] M. Navarro-Cía, M. Beruete, I. Campillo, and M. Sorolla, "Beamforming by Left-Handed Extraordinary Transmission Metamaterial bi-and plano-concave lens at millimeter-waves," IEEE Trans. Antennas Propag., vol. 59, no. 6, pp. 2141-2151, 2011.

[13] M. Beruete, M. Navarro-Cía, M. Sorolla, and I. Campillo, "Negative refraction through an extraordinary transmission left-handed metamaterial slab," Phys. Rev. B, vol. 79, no. 19, pp. 195107-1-6, May 2009.

[14] G. Dolling, C. Enkrich, M. Wegener, C. M. Soukoulis, and S. Linden, "Simultaneous negative phase and group velocity of light in a metamaterial," Science, vol. 312, no. 5775, pp. 892-894, 2006.

[15] W. E. Kock, "Metal-Lens Antennas," Proc. IRE, vol. 34, pp. 828-836, 1946.

[16] V. Pacheco-Peña, B. Orazbayev, V. Torres, M. Beruete, and M. Navarro-Cía, "Ultra-compact planoconcave zoned metallic lens based on the fishnet metamaterial," Appl. Phys. Lett., vol. 103, no. 18, p. 183507, 2013.

[17] B. Orazbayev, V. Pacheco-Peña, M. Beruete, and M. Navarro-Cía, "Exploiting the dispersion of the double-negative-index fishnet metamaterial to create a broadband low-profile metallic lens," Opt. Express, vol. 23, no. 7, pp. 8555-8564, 2015.

[18] S. M. Stout-Grandy, A. Petosa, I. V Minin, O. V Minin, and J. Wight, "A Systematic Study of Varying Reference Phase in the Design of Circular Fresnel Zone Plate Antennas," IEEE Trans. Antennas Propag., vol. 54, no. 12, pp. 3629-3637, 2006.

[19] V. Pacheco-Peña, M. Navarro-Cía, B. Orazbayev, I. V Minin, O. V Minin, and M. Beruete, "Zoned fishnet lens antenna with reference phase for side-lobe reduction," IEEE Trans. Antennas Propag., vol. 63, no. 8, pp. 3710-3714, 2015.

[20] M. Beruete, M. Sorolla, M. Navarro-cía, and F. Falcone, "Extraordinary transmission and left-handed propagation in miniaturized stacks of doubly periodic subwavelength hole arrays," Opt. Express, vol. 15, no. 3, pp. 1107-1114, 2007.

[21] B. Orazbayev, M. Beruete, and M. Navarro-Cía, "Wood zone plate fishnet metalens," EPJ Appl. Metamaterials, vol. 2, p. 8, 2015. 